\title{
Kleine-Levin syndrome: a unique cause of fatigue in an athlete
}

\author{
C M J Conklin, J E Taunton
}

Br J Sports Med 2005;39:e7 (http://www.bjsportmed.com/cgi/content/full/39/2/e7). doi: 10.1136/bjsm.2004.011809

Kleine-Levin syndrome (KLS) is a rare disorder characterised, most notably, by periodic episodes of hypersomnolence and hyperphagia. Associated features of the disorder include a lack of concentration, mood changes, and anxiety. Laboratory tests may show slight changes in the electroencephalogram. However, clinical presentation and laboratory tests are normal during asymptomatic intervals. KLS most offen presents in adolescent males, with complete recovery by the 3 rd to 4 th decade of life. Possible precipitating factors include excessive workload, febrile illness, and respiratory infections. Presented is a classical case of KLS in an adolescent male athlete. The patient's history, complete laboratory results, and symptoms are discussed. Possible treatments for this disorder are also mentioned, along with diagnostic criteria.

K leine-Levin syndrome (KLS) most often presents in adolescent males. ${ }^{1}$ It is characterised by episodes of hypersomnolence and hyperphagia. Fifty eight justified cases have been identified ${ }^{1}$ since clinical delineation by Kleine and Levin in the early 20th century. ${ }^{2}{ }^{3}$ Complex psychiatric and neurological symptoms often lead to erroneous diagnoses.

\section{CASE REPORT}

A 15 year old male athlete presented with intermittent hypersomnolent attacks of 10-12 days (eight days to three weeks) recurring over nine years. Twelve evenly distributed episodes occurred in the first two years, three in year 3, and one in year 4 . For the next five years, episodes occurred every one to two months. Between episodes the patient was completely asymptomatic.

A fever of $39^{\circ} \mathrm{C}$ and pharyngitis preceded the initial attack. A few days later he became emotionally flat, could not concentrate, lacked energy, and slept for 20 hours/day for eight to nine days. The most remarkable feature was excessive, sound sleep, which included rapid eye movement. He was easily roused, awakened himself to void his bladder, but neglected grooming. He craved and impulsively ate sweets when briefly awake during the episode. His lack of emotion was punctuated by immature behaviour. He spoke in phrases. He did not initiate conversation and was slow to respond to questions. His speech was sometimes slurred. He was irritable and apprehensive about medical examinations, and was antisocial with visitors. The patient had vivid, structured dreams of snakes, sharks, and vacations with his "wife". Sexual dreams dominated the end of the episode. On the last day of the episode, he developed an intense body odour accompanied by perfuse axillary sweating. Suddenly he "snapped out if it" overnight. He then suffered insomnia for two to four days, during which time he felt euphoric, dazed, confused, lacked concentration, and his mind raced. He was emotional about his health, his muscles were weak, and his joints ached, impeding normal stair climbing. After the episode, he had difficulty recalling events that had occurred during the attack. Interestingly, a neuropsychological assessment during the episode revealed "acute confusion", which may interfere with memory encoding. The patient soon returned to school and sports.

Subsequent episodes were marked by the same symptoms, although less severe. He was slightly less detached and more verbal in later episodes. He also slept slightly fewer hours, and improved gradually throughout subsequent episodes.

His behaviour for the two days preceding episodes was remarkably consistent. He would have a sore throat, feel disoriented, and anxious. He socially withdrew and could not complete verbal sentences. His concentration was impaired and he could not perform simple maths. Alcohol intake preceded some episodes, and exercise was believed to be a trigger. His involvement in exhaustive sports as a healthy individual contradicts this.

The patient was given lorazepam during an attack to which he developed a paradoxical reaction. During other attacks he was given amantadine, desipramine, and trifluoperazine. These drugs did not have any notable effects.

Neurologists, immunologists, psychiatrists, psychologists, and sports medicine specialists examined the patient both during and between episodes. Blood pressure and pulse were normal. Pupils were sometimes dilated during attacks. Body temperature was often $35^{\circ} \mathrm{C}$. All central and peripheral nervous system tests, including motor and sensory functions, were normal. Computed tomography scans, brain magnetic resonance imaging scans, and cerebral blood flow images were normal. Blood tests for urea, creatine, fasting glucose, calcium, magnesium, lactate, bilirubin, phosphorus, and liver enzymes were normal. Morning and evening cortisol concentrations were normal, as were thyroid hormone (triiodothyronine and free thyroxine) and thyroid stimulating hormone concentrations. Amino acid serum screen showed slightly raised immuno $N$-butyric acid and valine. Other tests for inborn metabolic errors were normal. During one episode, IgM was raised, and activated $\mathrm{T}$ cells and $\operatorname{IgE}$ concentrations were massively increased at $3039 \mu \mathrm{g} / \mathrm{l}$. On two occasions, he had a raised Epstein-Barr IgM to IgG ratio indicative of acute mononucleosis. White cell counts were consistently normal. Porphyrin and haemoglobin tests were normal. Several spinal fluid analyses during both symptomatic and asymptomatic periods showed normal concentrations of protein, glucose, and albumin and white and red blood cell counts. IgG synthesis within the cerebrum and spinal cord was never increased. A muscle biopsy test for mitochondrial myopathy was negative. Extensive psychiatric investigations revealed no disorder. Psychological examination during an attack revealed an acute concentration deficit especially in nonverbal reasoning.

Between episodes he excelled in sports and maintained honour roll standing throughout high school and university. He functioned well under high stress. His family medical history was unremarkable. He is now healthy and has been 
Table 1 Diagnostic features of Kleine-Levin syndrome

\begin{tabular}{llll}
\hline Classical form & Associated features & Atypical forms & Exclude from diagnosis of KLS \\
\hline Onset in adolescence & $\begin{array}{l}\text { Prominent mental and } \\
\text { mood changes } \\
\text { Sometimes hypersexuality } \\
\text { in attacks } \\
\text { Episodic hypersomnia } \\
\text { findings between attacks }\end{array}$ & $\begin{array}{l}\text { One or more main } \\
\text { symptoms absent }\end{array}$ & Cases with symptom inversion \\
Excessive eating & Menstrual hypersomnolence \\
Spontaneous recovery & Male:female ratio 4:1 & $\begin{array}{l}\text { Organic hypothalamic } \\
\text { pathology } \\
\text { Psychiatrically determined } \\
\text { cases }\end{array}$ \\
\hline
\end{tabular}

asymptomatic for more than five years. No residual deficits are notable.

\section{DISCUSSION}

This case study exemplifies classic KLS. The patient was male and began experiencing episodes in adolescence. He displayed hypersomnia and megaphagia during episodes, as well as other typical KLS behaviours. ${ }^{1}$ He spontaneously recovered from episodes, functioned normally between, ${ }^{4}$ and was extensively examined to rule out other disorders. ${ }^{1}$ The average KLS attack lasts five to seven days. ${ }^{1}$ Durations can range from a few days to several weeks with prolonged attacks being unusual. ${ }^{1}$ Episode frequency ranges from one a month to one a year. ${ }^{1}$ Disinhibited sexual thoughts and behaviour occur in $23 \%$ of male patients and $20 \%$ of female patients. ${ }^{1}$ Motor unrest can occur during awake periods ${ }^{3}$ along with grandiose behaviour and auditory and visual hallucinations. ${ }^{5}$ Although euphoria and mood elevation often follow an attack, depression and suicidal tendencies may also occur. ${ }^{6}$

The exact cause is unknown. Several precipitating factors have been reported. Febrile illnesses have preceded initial attacks. ${ }^{4}$ Respiratory infections, ${ }^{7}$ sore throats, tonsillitis, ${ }^{3}$ and other acute illnesses often antedate subsequent attacks. Stress $^{1}$ and drunkenness ${ }^{8}$ are other possible precipitating factors.

Laboratory investigations are usually normal between and during attacks. ${ }^{137-9}$ Table 1 provides an outline for preliminary diagnosis. Differential disorders include symptom inversion, such as insomnia instead of hypersomnia, menstrual related hypersomnia often with cyclical oedema, psychiatrically determined cases or cases with evidence of hypothalamic pathology. ${ }^{1}$

Polysomnographic recordings should investigate daytime sleepiness to eliminate narcolepsy, circadian rhythm disturbances, chronic hypersomnia, sleep apnoea, and pickwickian syndrome. ${ }^{5}$

Psychiatric problems such as schizophrenia, hysteria, depression, and mania should also be ruled out. ${ }^{5}$ These disorders may be discounted when DSM-III criteria are not met, when the patient and his/her family's psychiatric history is negative, and when the onset of episodes are brief.5 Hypothalamic dysfunction has been targeted as a symptom source. However, concentrations of adrenocorticotrophic hormone, growth hormone, and thyroid releasing hormone in patients with KLS are inconsistent, confusing the origin of hypothalamic abnormality. ${ }^{10}$

KLS is benign and attacks usually cease by the 3rd-4th decade. $^{1}$ However, it is a debilitating disorder during symptomatic periods. Amphetamines and other stimulants, such as methylphenidate and ephedrine, have reduced attack severity and frequency in some patients. ${ }^{6}$ Stimulants have also prevented subsequent attacks. Tricyclic antidepressants, anticonvulsants, and antipsychotic drugs have sedating side effects and generally are not useful. ${ }^{5}$ Lithium carbonate has prevented future attacks, ${ }^{9}$ or caused improvement in some patients, ${ }^{7}$ but has had no effect on others. ${ }^{9}$ Patients for whom lithium treatment has been successful often have periodic hypersomnia but not morbid hunger. ${ }^{7}$ These cases may be considered atypical and seemingly associated with cyclical depression. ${ }^{1}$ To improve treatment and identification of this disorder, further research is required to clarify its aetiology and pathophysiology.

\section{Authors' affiliations}

C M J Conklin, J E Taunton, University of British Columbia, Vancouver, BC, Canada

Conflict of interests: none declared

Correspondence to: Mr Conklin, Faculty of Medicine, University of British Columbia, 317-2194 Health Sciences Mall, Vancouver, BC V6T 1Z3, Canada; conklin@interchange.ubc.ca

Accepted 11 May 2004

\section{REFERENCES}

1 Parkes JD. Kleine-Levin syndrome. In: Sleep and its disorders. London: WB Saunders, 1985:314-20.

2 Kleine W. Periodische Schlafsucht. Monatsschr Psychiatr Neurol 1925;57:285-320.

3 Levin M. Periodic somnolence and morbid hunger: a new syndrome. Brain 1936;58:494-515.

4 Wygnanski T, Kokia E, Barak P, et al. The sleeping aviator: aeromedical disposition of Kleine-Levin syndrome. Aviat Space Environ Med 1996:67:61-2.

5 Orlosky MJ. The Kleine-Levin syndrome: a review. Psychosomatics 1982;23:609-21.

6 Gallinek A. The Kleine Levin syndrome: hypersomnia, bulimia and abnormal mental states. World Neurol 1962;3:235-41.

7 Goldberg MA. The treatment of Kleine-Levin syndrome with lithium. Can J Psychiatry 1983;28:491-2.

8 Critchley M. Periodic hypersomnia and megaphagia in adolescent males. Brain 1962;85:628-56.

9 Pike M, Stores G. Kleine-Levin syndrome: a cause of diagnostic confusion. Arch Dis Child 1994;71:355-7.

10 Godath N, Dickerman Z, Bechar M. Episodic hormone secretion during sleep in Kleine-Levin syndrome: evidence for hypothalamic dysfunction. Brain Dev 1987;9:309-15.

\section{COMMENTARY}

$\mathrm{K}$ lein-Levin syndrome is a rare and unusual neurological syndrome that would be out of the experience of most, if not all, sports physicians. This case illustrates the difficulties in the diagnosis of such a case in an adolescent athlete. Fortunately, most cases recover spontaneously with little in the way of long term sequelae.

P McCrory

University of Melbourne, Centre for Health, Exercise and Sports Medicine, Melbourne, Victoria 3010, Australia; pmccrory@compuserve.com 